\title{
Utilization of risk-based predictive stability within regulatory submissions; industry's experience
}

\author{
Megan McMahon ${ }^{1 *} \mathbb{D}$, Helen Williams ${ }^{2}$, Elke Debie ${ }^{3}$, Mingkun Fu ${ }^{4}$, Robert Bujalski ${ }^{4}$, Fenghe Qiu ${ }^{5}$, Yan Wu ${ }^{6}$, \\ Hanlin $\mathrm{Li}^{7}$, Jin Wang ${ }^{8}$, Cherokee Hoaglund-Hyzer ${ }^{9}$ and Donnie Pulliam ${ }^{10}$
}

\begin{abstract}
Risk-Based Predictive Stability (RBPS) tools, such as the Accelerated Stability Assessment Program (ASAP) and other models, are used routinely within pharmaceutical development to quickly assess stability characteristics, especially to understand mechanisms of degradation. These modeling tools provide stability insights within weeks that could take months or years to understand using long-term stability conditions only. Despite their usefulness, the knowledge gained through these tools are not as broadly used to support regulatory filing strategies. This paper aims to communicate how industry has used RBPS data to support regulatory submissions and discuss the regulatory feedback that was received.
\end{abstract}

Keywords: Risk-based predictive stability, RBPS, Clinical shelf-life, Predictive, Regulatory feedback

\section{Introduction}

In 2015, the International Consortium for Innovation and Quality in Pharmaceutical Development (IQ) launched a working group to focus on the use of RiskBased Predictive Stability (RBPS) tools to optimize pharmaceutical development. The working group has approximately 50 members from 18 companies across the pharmaceutical industry. The working group conducted an industry survey to understand sponsor companies' experiences using RBPS tools (Williams et al. 2017). The survey indicated that RBPS tools were being utilized in a variety of applications across the development continuum. A key learning was that of all the surveyed companies utilizing RBPS tools, approximately $55 \%$ of them

\footnotetext{
* Correspondence: megan.mcmahon@pfizer.com

This paper was developed with the support of the International Consortium for Innovation and Quality in Pharmaceutical Development (IQ, www. iqconsortium.org). IQ is a not-for-profit organization of pharmaceutical and biotechnology companies with a mission of advancing science and technology to augment the capability of member companies to develop transformational solutions that benefit patients, regulators and the broader research and development community

${ }^{1}$ Pfizer Inc., Eastern Point Road, Groton, CT 06340, USA

Full list of author information is available at the end of the article
}

were leveraging the data in a regulatory capacity. Of those that reported utilization of RBPS data in regulatory submissions, the level of experiences exceeded 100 submissions in total from clinical development through registration. This paper provides a more in-depth look at these submissions and the regulatory feedback that was received. The ideas shared in this document reflect information shared during the 2018 AAPS and IQ Joint Short Course entitled "Science and Risk-Based Stability Strategies: Applications of Predictive Tools."

The International Council for Harmonization (ICH) first recommended the adoption of Guideline Q1A (Stability Testing of New Drug Substances and Products) in 2000 (ICH 2003). Since that time, significant advances in the science of stability have occurred. Improved modeling tools coupled with appropriate protocols now enable confident stability predictions without long-term data (Waterman and Adami 2005; Waterman et al. 2007; Oliva et al. 2012; Wicks et al. 2018; Williams et al. 2018). Although these tools do not replace the traditional long-term and accelerated studies that are described in ICH Q1A, they enhance drug development by 
providing an increased understanding of the attributes that influence stability earlier in development. These approaches are well aligned with the science and riskbased approaches detailed in ICH Q8--Q11 (ICH 2009; ICH 2005; ICH 2008; ICH 2012) and have been termed Risk-Based Predictive Stability (RBPS) (Williams et al. 2017). Companies are utilizing these RBPS tools to enable the development of medicines (Freed et al. 2016).

The survey indicated that the majority of the regulatory experience has been in the early clinical development phase (Williams et al. 2017). Based on this, the working group published a proposed regulatory template to standardize how RBPS data can be reported in a regulatory filing (Stephens et al. 2018) to support an initial clinical shelf life. This paper is intended to expand on the regulatory template publication by sharing diverse case studies of instances where RBPS tools have been used to support regulatory filings. The case studies illustrate how RBPS tools can be used throughout all stages of development. The industry survey indicated that most companies using RBPS tools are utilizing Accelerated Stability Assessment Program (ASAP) modeling (Williams et al. 2017). The case studies reflect the predominant use of ASAP; however, other predictive models could support similar regulatory strategies.

The presented case studies are summarized in Table 1. The majority focus on early clinical development, which reflects how industry most commonly uses RBPS tools in regulatory submissions. Where RBPS is used to predict a retest date/shelf life, the predictions are always supported by a $95 \%$ confidence interval. However, most companies take a conservative approach to retest/shelflife dating and assign a retest/shelf-life shorter than the prediction supports. Typically, if RBPS data alone is used to establish the retest/shelf-life, a $1-2$ year maximum is applied.

The case studies are presented in order of the phase of development. Most of these case studies utilize RBPS tools to predict degradation, but RBPS tools can also be used to predict dissolution. Case studies were provided by eight pharmaceutical companies. Additionally, the collective regulatory experiences from 5 companies were pooled and analyzed with respect to global regulatory acceptance of RBPS strategies.

\section{Case studies - phase 1}

Case study 1: setting an initial retest period for drug substance and an initial shelf-life of the drug product Accelerated Stability Assessment Program (ASAP) studies on drug substance and drug product were performed in 2014 to support a first-in-human (FIH) clinical trial with an oral solution. The 14-day ASAP study for the drug substance included seven temperature and humidity conditions ranging from $40{ }^{\circ} \mathrm{C} / 75 \% \mathrm{RH}$ to $80{ }^{\circ} \mathrm{C} / 10 \% \mathrm{RH}$. The ASAP study for the drug product (oral solution) was one month long, and was performed with five different temperatures ranging from $25^{\circ} \mathrm{C}$ to $70^{\circ} \mathrm{C}$. In both cases, assay and chemical degradation were modeled. For the oral solution, the ASAP was performed in closed vials and for the drug substance, the ASAP was performed in an open dish.

The ASAP predictions and 1-month of stability data at the proposed long-term storage condition on the drug substance were used to support an initial retest period of 12 months, although the modeling predicted a retest period of at least 3 years. For the oral solution, a shelflife of 6 months when stored at $2-8^{\circ} \mathrm{C}$ was proposed based on ASAP data only since no out-of-specification test results were predicted for at least 1 year at $5{ }^{\circ} \mathrm{C}$ and at least 6 months at $25^{\circ} \mathrm{C} / 60 \% \mathrm{RH}$ storage conditions. In the IMPD, a commitment was made to perform traditional long-term and accelerated stability monitoring on both drug substance and drug product and the stability protocols were included. The submission was filed in Belgium in 2014 and was accepted without any related regulatory queries. The ASAP predictions were subsequently confirmed by the available long-term stability data.

\section{Case study 2: leveraging RBPS to support shelf-life for a new strength of an existing tablet formulation}

In 2015, a Phase 1 clinical trial was proposed requiring a new lower strength version of an existing tablet formulation. The existing tablets were known to be chemically stable with a 5-year shelf-life based on five years of stability data at $25^{\circ} \mathrm{C} / 60 \% \mathrm{RH}$ and significant product understanding and development knowledge. The new low strength tablet was formulated utilizing a common granule with the existing tablets and therefore expected to also be chemically stable. An ASAP study incorporating five temperature and humidity conditions from $50{ }^{\circ} \mathrm{C} /$ $75 \% \mathrm{RH}$ to $70{ }^{\circ} \mathrm{C} / 75 \% \mathrm{RH}$ for up to 4 weeks was performed using a crushed tablet, as the worst-case scenario due to increased surface area. Chemical degradation was modeled based on one main degradant as the shelf-life limiting attribute (SLLA). Predictions did not consider packaging (worst case).

The ASAP predictions were used to support a 3year shelf-life claim, for the new low strength tablet, along with 3 months long-term stability data on the new strength and previous stability data on the existing higher strength formulations. The submission was filed in the USA, UK, France, Italy, Turkey, Egypt, Lebanon and Kenya in 2015 and was accepted without any related regulatory queries. The ASAP predictions were subsequently confirmed by the available long-term stability data. 
Table 1 Overview of case studies

\begin{tabular}{|c|c|c|c|c|c|c|}
\hline \multirow{2}{*}{$\begin{array}{l}\text { Case } \\
\text { study } \\
\text { no. }\end{array}$} & \multirow[t]{2}{*}{ Description } & \multirow[t]{2}{*}{ Phase } & \multirow[t]{2}{*}{ Material } & \multirow{2}{*}{$\begin{array}{l}\text { Submission Content in } \\
\text { Addition to Predictive }_{\text {Data }^{\mathrm{a}}}\end{array}$} & \multicolumn{2}{|l|}{ Territories } \\
\hline & & & & & Accepted & Questioned \\
\hline 1 & $\begin{array}{l}\text { Setting initial retest period and } \\
\text { shelf-life }\end{array}$ & Phase 1 & $\begin{array}{l}\text { Drug } \\
\text { substance } \\
\text { and oral } \\
\text { solution }\end{array}$ & $\begin{array}{l}\text { Limited long-term data } \\
\text { for drug substance }\end{array}$ & Belgium & \\
\hline 2 & $\begin{array}{l}\text { Setting shelf-life of formulation } \\
\text { variant }\end{array}$ & Phase 1 & Tablet & Limited long-term data & $\begin{array}{l}\text { USA, UK, France, Italy, Turkey, Egypt, } \\
\text { Lebanon, Kenya }\end{array}$ & \\
\hline 3 & $\begin{array}{l}\text { Setting shelf-life of formulation } \\
\text { variant }\end{array}$ & Phase 1 & Capsule & Limited long-term data & USA & \\
\hline 4 & $\begin{array}{l}\text { Setting initial retest period and } \\
\text { shelf-life }\end{array}$ & Phase 1 & $\begin{array}{l}\text { Drug } \\
\text { substance } \\
\text { and IV } \\
\text { solution }\end{array}$ & None & $\begin{array}{l}\text { Netherlands, Germany (drug } \\
\text { substance) }\end{array}$ & $\begin{array}{l}\text { UK, } \\
\text { Germany } \\
\text { (drug } \\
\text { product) }\end{array}$ \\
\hline 5 & $\begin{array}{l}\text { Setting initial retest period and } \\
\text { shelf-life }\end{array}$ & Phase 1 & $\begin{array}{l}\text { Drug } \\
\text { substance } \\
\text { and tablet }\end{array}$ & None & USA & UK \\
\hline 6 & Setting initial shelf-life & Phase 1 & Tablet & None & Belgium, Moldova, Bulgaria, Georgia & $\begin{array}{l}\text { France, } \\
\text { Spain }\end{array}$ \\
\hline 7 & Setting initial shelf-life & Phase 1 & $\begin{array}{l}\text { Powder for } \\
\text { oral } \\
\text { solution } \\
\text { and tablet }\end{array}$ & None & & Germany \\
\hline 8 & $\begin{array}{l}\text { Setting shelf-life of formulation } \\
\text { variant }\end{array}$ & Phase 1 & Capsule & None & USA, Spain, France & \\
\hline 9 & Setting initial shelf-life & Phase 2 & Tablets & None & USA, Belgium & \\
\hline 10 & Setting initial shelf-life & Phase 2 & $\begin{array}{l}\text { Modified } \\
\text { release } \\
\text { formulation }\end{array}$ & None & USA, Belgium & \\
\hline 11 & $\begin{array}{l}\text { Setting shelf-life of formulation } \\
\text { variant }\end{array}$ & Phase 2 & Tablet & None & USA, Spain, France & Germany \\
\hline 12 & $\begin{array}{l}\text { Justify storage condition for drug } \\
\text { substance with a new process, } \\
\text { and predict shelf-life for a new } \\
\text { formulation }\end{array}$ & Phase 2 & $\begin{array}{l}\text { Drug } \\
\text { Substance } \\
\text { and Tablet }\end{array}$ & $\begin{array}{l}\text { Limited long-term data } \\
\text { for drug substance, Lim- } \\
\text { ited long-term data for } \\
\text { drug product }\end{array}$ & USA & \\
\hline 13 & $\begin{array}{l}\text { Setting shelf-life of formulation } \\
\text { variant }\end{array}$ & Phase 2 & Tablet & Limited long-term data & UK (but with reduced shelf-life) & \\
\hline 14 & $\begin{array}{l}\text { Impact of drug product process } \\
\text { change on shelf-life }\end{array}$ & Phase 3 & Tablet & Limited long-term data & $\begin{array}{l}\text { USA, Canada, Russia, Taiwan, Poland, } \\
\text { Ukraine, Japan }\end{array}$ & \\
\hline 15 & $\begin{array}{l}\text { Setting shelf-life of formulation } \\
\text { variant }\end{array}$ & Phase 3 & Capsule & $\begin{array}{l}\text { Supportive information } \\
\text { from previous } \\
\text { formulations }\end{array}$ & $\begin{array}{l}\text { Belgium, Brazil, Canada, China, India, } \\
\text { Denmark. Finland, France, Ireland, } \\
\text { Italy, Netherlands, Portugal, Spain, } \\
\text { Taiwan, Turkey }\end{array}$ & $\begin{array}{l}\text { UK, Korea, } \\
\text { Germany }\end{array}$ \\
\hline 16 & $\begin{array}{l}\text { Justification of strategy for water } \\
\text { testing on stability }\end{array}$ & Registration & Tablet & $\begin{array}{l}\text { Predictive dissolution } \\
\text { model }\end{array}$ & USA, EU & \\
\hline 17 & $\begin{array}{l}\text { Justification of degradant end of } \\
\text { life specification }\end{array}$ & Registration & Tablet & Limited long-term data & $\begin{array}{l}\text { USA, EU, Canada, Australia, Asia } \\
\text { Pacific and Latin America }\end{array}$ & \\
\hline
\end{tabular}

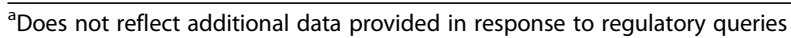

\section{Case study 3: leveraging RBPS to support a change in the capsule shell}

In late 2016, a Phase 1 capsule formulation was reformulated to change from a gelatin capsule shell to a hydroxypropyl methyl cellulose (HPMC) capsule shell. An ASAP study was performed to predict a shelf-life for the new encapsulated formulation using a representative batch. The 8-week ASAP study incorporated six temperature and humidity conditions ranging from $50{ }^{\circ} \mathrm{C} / 30 \% \mathrm{RH}$ to $70^{\circ} \mathrm{C} / 75 \% \mathrm{RH}$ and chemical degradation was modeled based on the formation of three degradants, to identify the shelf-life limiting degradant.

The ASAP predictions were submitted to the USA in 2017 with one month of long-term stability data for the HPMC capsules to support a 12-month shelf-life under ambient conditions. This was accepted with no related 
regulatory queries. The ASAP predictions were subsequently confirmed by the available long-term stability data.

\section{Case study 4: setting an initial retest period for drug substance and an initial shelf-life for a parenteral drug product}

During 2017, ASAP studies were performed for the first Phase 1 submission for a new drug substance and the drug product formulation, an IV solution. The ASAP study performed for the drug substance utilized six temperature and humidity conditions ranging from $50{ }^{\circ} \mathrm{C} / 75 \%$ RH to $80^{\circ} \mathrm{C} / 30 \% \mathrm{RH}$. No degradation was observed during the 12-week drug substance ASAP study, so no modeling was performed. A similar range of temperatures were used for the drug product 6-week ASAP study, using a representative batch, and the data used to predict an initial shelf-life based on formation of the main degradant.

The ASAP data for the drug substance and drug product were presented in the application with predicted degradation for the drug product. The ASAP data were used to support a 12-month retest period for the drug substance under ambient conditions and a 12-month for the drug product at $5{ }^{\circ} \mathrm{C}$. Commitments to initiate traditional long-term and accelerated stability studies for both the drug substance and drug product were made, but no long-term stability data were presented. The submission was filed in the UK, Germany and the Netherlands. It was accepted with no related queries in the Netherlands. The drug substance retest period was accepted by Germany, but the drug product shelf-life claim was questioned and a 6-month shelf-life was suggested. In response, the 1 and 3-month long-term stability data for the drug product was presented to support the 12-month shelf-life claim. The MHRA requested long-term stability data for both drug substance and drug product, which was subsequently provided. In the responses to both Germany and the UK, the 1 and 3month long-term stability data for the drug product was overlaid with the ASAP predictions at both $5{ }^{\circ} \mathrm{C}$ and $25^{\circ} \mathrm{C}$ to demonstrate the accuracy of the predictions and the applications were approved. The ASAP predictions were subsequently confirmed by the available long-term stability data.

\section{Case study 5: setting an initial retest period for drug substance and an initial shelf-life for drug product}

ASAP studies were performed during 2017 to support the initial Phase 1 submission for a new drug substance and drug product tablet formulation, using representative batches. The 6-week ASAP study incorporated six temperature and humidity conditions ranging from $50{ }^{\circ} \mathrm{C} / 75 \% \mathrm{RH}$ to $80{ }^{\circ} \mathrm{C} / 30 \% \mathrm{RH}$ for the drug substance and drug product. Minimal degradation was observed in the drug substance ASAP study, so modeling was not possible. Shelf-life predictions were performed for the drug product based on the main degradant.

The ASAP data for drug substance and drug product were presented in the submission with predicted degradation for the drug product. The ASAP data supported an initial 12-month retest period for the drug substance and an initial 12-month shelf-life for the drug product under ambient conditions. Commitments to complete traditional long-term and accelerated stability studies for both the drug substance and drug product were stated, but no long-term stability data were presented. The submission was filed in the USA and UK. The US FDA had no related queries, but UK MHRA requested 3 months long-term stability data for both the drug substance and drug product. The requested data was supplied resulting in an agreement with the 12-month shelf-life and no delay to the clinical study start. The ASAP predictions were subsequently confirmed by the available long-term stability data.

\section{Case study 6: setting an initial shelf-life for drug product}

In support of a Phase 1 clinical trial, a 30-day ASAP study was performed applying 6 different temperature and humidity conditions ranging from $40{ }^{\circ} \mathrm{C} / 75 \% \mathrm{RH}$ to $70{ }^{\circ} \mathrm{C} / 75 \% \mathrm{RH}$, on newly formulated tablets. During the ASAP study, no degradation was observed, so modeling was not possible. A shelf-life of 12 months was proposed for the tablets. The submission was filed in 2015 to Belgium, Spain, France, Moldova, Bulgaria and Georgia and was accepted in all countries, except for France and Spain where additional long-term data (3 months) was requested to approve the 12-month shelf-life. The ASAP predictions were subsequently confirmed by the available long-term stability data.

\section{Case study 7: setting an initial retest period for drug substance and an initial shelf-life for multiple drug products}

A new Phase 1 clinical trial was proposed using a powder for oral solution (PfOS), consisting of drug substance in a bottle formulation and a tablet formulation. A 12month shelf-life was required for both formulations to enable the clinical study. ASAP studies at four temperature and humidity conditions ranging from $60{ }^{\circ} \mathrm{C} / 50 \% \mathrm{RH}$ to $70{ }^{\circ} \mathrm{C} / 75 \% \mathrm{RH}$ were conducted for up to three weeks on the drug substance and the tablet. No degradation was observed. The ASAP data were submitted to Germany in 2014 to request an initial 12-month shelf-life for the PfOS and the tablet. The submission also included a commitment to monitor clinical batches using a traditional long-term and accelerated stability protocol. No long-term data were provided in the initial submission. 
The Agency responded that "The submitted stability data are inadequate for demonstrating a shelf-life of 12 months for both investigational preparations. Additional results should be submitted which at least demonstrate the stability of the active ingredient and the tablets after 3 months at $40^{\circ} \mathrm{C} / 75 \% \mathrm{RH}$ ". At the time the query was received, the 3-month results on the tablet were available and submitted together with 3-month drug substance data at $40{ }^{\circ} \mathrm{C} / 75 \% \mathrm{RH}$. No further queries were received from the Agency and the 12-month shelf-life was approved. The ASAP predictions were subsequently confirmed by the available long-term stability data.

\section{Case study 8: setting an initial shelf-life for a new formulation for an ongoing clinical study}

In 2013, a formulation was changed from an oral solution to a capsule for an ongoing Phase 1 clinical trial. At the time of submission of the new formulation, no longterm stability data were available. A 14-day ASAP study incorporating five temperature and humidity conditions ranging from $40{ }^{\circ} \mathrm{C} / 75 \% \mathrm{RH}$ to $70{ }^{\circ} \mathrm{C} / 50 \% \mathrm{RH}$ was performed. Assay and chemical degradation were modeled. Packaging predictions were also included.

The ASAP predictions were used to support an initial 12-month shelf-life for the new capsule formulation. The submission included ASAP data with a commitment to perform long-term stability on a representative capsule batch. The application was approved in the USA, France, and Spain in 2013. No regulatory queries were received on the proposed shelf-life. The ASAP predictions were subsequently confirmed by the available longterm stability data.

\section{Case studies - phase 2 \\ Case study 9: setting an initial shelf-life for multiple tablet strengths (not common blend)}

Clinical studies for a tablet formulation at three strengths manufactured at varying drug loads were planned for the USA and Belgium in 2016. The submissions did not include long-term stability data but did provide a commitment to place representative clinical batches of each strength on long-term stability. An ASAP study was performed on a development batch of compacts for the strength with the highest excipient to drug ratio, maximizing the potential for drug substance degradation.

The ASAP experiment was run for 14 days in open containers incorporating six temperature and humidity conditions ranging from $50{ }^{\circ} \mathrm{C} / 75 \% \mathrm{RH}$ to $80{ }^{\circ} \mathrm{C} / 40 \%$ $\mathrm{RH}$. The results included levels for two primary degradants. The data was modeled and predicted that room temperature storage may not provide a suitable shelflife, but a shelf-life of 12 months could confidently be set for all three strengths when stored at $5{ }^{\circ} \mathrm{C}$. These data and predictions were submitted to both health authorities. No queries were received. Since the initial submissions, long-term data has supported the storage of the supplies under refrigeration and room temperature.

\section{Case study 10: setting an initial shelf-life for a modified release drug product}

A modified-release (MR) formulation at two strengths (the strengths were composed of different levels of drug substance to produce a common tablet weight) was introduced into clinical studies in the USA and Belgium. At the time of this 2015 submission, no long-term data was available, and the initial shelf-life was supported by an ASAP study.

The ASAP study was run on both tablet strengths for 60 days using eight temperature and humidity conditions, ranging from $30{ }^{\circ} \mathrm{C} / 5 \% \mathrm{RH}$ to $60{ }^{\circ} \mathrm{C} / 5 \% \mathrm{RH}$ and $50{ }^{\circ} \mathrm{C} / 52 \% \mathrm{RH}$. A single degradant was identified as the SLLA and was modeled to predict that the MR tablets would be stable for at least 12 months when stored at $25^{\circ} \mathrm{C}$. An initial shelf-life of 12 months was proposed. The results of the ASAP study were submitted along with a commitment to place a representative clinical batch of each strength on long-term and accelerated stability. No related regulatory queries were received. The ASAP predictions were subsequently confirmed by the available long-term stability data.

\section{Case study 11: setting an initial shelf-life for a new formulation introduced into an ongoing clinical study}

In 2017, clinical application amendments were filed to introduce an additional tablet formulation. Two tablet strengths with a different formulation were already approved for clinical use. A different strength was proposed for the new formulation and no long-term data was available for the clinical amendments.

A 4-week ASAP study was conducted to support the initial clinical shelf-life for the new formulation and results were provided in the regulatory submission. The study was conducted on a development strength tablet $(0.25 \mathrm{mg}$ instead of the proposed clinical strength of 15 $\mathrm{mg})$. The development strength $(0.25 \mathrm{mg})$ contained a higher excipient to drug ratio than the proposed clinical strength, thereby providing a worst-case assessment for degradation. They were stored in open glass bottles and exposed to eleven separate temperatures, humidities and durations. The conditions varied from $50{ }^{\circ} \mathrm{C} / 75 \% \mathrm{RH}$ to $80{ }^{\circ} \mathrm{C} / 40 \% \mathrm{RH}$ and $70{ }^{\circ} \mathrm{C} / 75 \% \mathrm{RH}$.

The stability samples were subsequently evaluated for appearance and concentrations of the main degradant. The main degradant was used to predict the shelf-life and predictions supported an initial shelf-life of at least 12 months. 
This application was filed to the USA, Spain, Germany and France. For Spain, the USA and France, an initial shelf-life of 12 months was accepted without related regulatory queries. Germany queried the assignment of a 12-month shelf-life without long-term data. In response, 3 months of long-term data were provided at the proposed storage conditions and accelerated conditions $\left(40{ }^{\circ} \mathrm{C} / 75 \% \mathrm{RH}\right)$ and the shelf-life was approved. The ASAP predictions align with the available long-term stability data.

\section{Case study 12: justification of storage conditions for drug substance and setting an initial shelf-life for drug product} ASAP studies on drug substance and drug product were performed to support a Phase 2 clinical program in 2018. The drug substance was manufactured via a new process and a new drug product was introduced. In both cases, the studies were performed on representative development batches in an open dish configuration, and chemical degradation kinetics were modeled on the major degradant. The 42-day ASAP study for the drug substance included 5 temperature and humidity conditions ranging from $50{ }^{\circ} \mathrm{C} / 80 \% \mathrm{RH}$ to $80{ }^{\circ} \mathrm{C} / 0 \% \mathrm{RH}$. For drug product, the 100 day ASAP study was performed based on 5 different temperature and humidity conditions ranging from $50{ }^{\circ} \mathrm{C} / 60 \% \mathrm{RH}$ to $70{ }^{\circ} \mathrm{C} / 10 \% \mathrm{RH}$. The long study duration was due to the need to use less stressful conditions because of potential form changes at higher temperatures. The ASAP predictions and 9month long-term stability on clinical batches of drug substance were used to justify refrigerated storage condition. For drug product, the ASAP model was used to support a predicted 2-year shelf-life. The ASAP results were submitted to FDA, together with 6 months of stability on developmental batches and a commitment to perform long-term stability on the clinical batches. This risk-based stability approach was accepted by the agency without stability data on clinical batches. Long-term stability studies on the clinical batches are ongoing. The ASAP predictions align with the available long-term stability data.

\section{Case study 13: setting an initial shelf-life of a drug product} In 2018 a new drug product formulation was planned for use in a Phase 2 relative bioavailability (rBA) study in the UK. Subsequently, the same formulation would be used in Phase 3 studies. A 28-day ASAP open-dish study was conducted on a representative lot. Experiments included 9 temperature and humidity conditions ranging from $30{ }^{\circ} \mathrm{C}$ to $80{ }^{\circ} \mathrm{C}$ and $11 \% \mathrm{RH}$ to $82 \% \mathrm{RH}$. Chemical degradation was modeled based on a major degradant. The ASAP model predicted acceptable stability in the clinical package for at least 1.6 years at ambient storage conditions.
The ASAP results were submitted to support a 12month shelf-life for the new formulation. One month of long-term stability data on a representative batch was submitted with a commitment to continue the confirmatory long-term stability study. A commitment was also made to run a confirmatory long-term stability study on a clinical batch through the proposed shelf-life. The UK reviewer did not query the approach but reduced the shelf-life to 6 months as part of the IMPD approval letter. There was no opportunity to provide additional stability data during the review. The ASAP predictions align with the available long-term stability data.

\section{Case studies - phase 3 \\ Case study 14: re-assessment of shelf-life after a drug product process change}

During the Phase 3 development of a tablet formulation in 2016, a process change from wet granulation to roller compaction was required. The product was known to be chemically stable, with two years long- term stability data on the wet granulation product. An 8-week ASAP study was performed on a representative batch of the roller compacted formulation, utilizing six temperature and humidity conditions ranging from $50{ }^{\circ} \mathrm{C} / 75 \% \mathrm{RH}$ to $80^{\circ} \mathrm{C} / 30 \% \mathrm{RH}$. No degradation was observed in the ASAP study, demonstrating the chemical stability of the product. A reduction in the rate of dissolution was observed in tablets exposed to high humidity. This trend was consistent with previous studies of the wet granulation formulation; therefore, the tablets were stored with desiccant. The results from both studies were used to demonstrate equivalence between the two formulations with respect to stability attributes.

The ASAP and dissolution data for the roller compaction formulation was presented in the regulatory submission to support a 2-year shelf-life alongside one month of long-term stability data and 2-year stability data on the wet granulation formulation. The submission was filed in the US, Canada, Russia, Taiwan and Poland and accepted with no related regulatory queries. Long-term data subsequently confirmed that the assigned shelf-life was appropriate.

During 2017 a lower strength tablet of the same roller compaction formulation (common blend) was required for clinical studies. The ASAP and dissolution studies were repeated for the low strength formulation and similar results obtained. The data was used to demonstrate equivalence with the higher strength formulation and the wet granulation formulation. The ASAP and dissolution data were presented in the regulatory submission in the absence of long-term data on the low strength formulation, but alongside 6 months stability data on the higher strength formulation. A commitment was made to set down long-term stability studies for the low 
strength formulation in the submission. It was filed in Ukraine, Russia, Canada, US, Taiwan, Poland and Japan and was accepted without related regulatory queries. The ASAP predictions were subsequently confirmed by the available long-term stability data.

\section{Case study 15: setting an initial shelf-life for a new drug product formulation}

In 2010, IND and IMPD applications were filed globally (Belgium, Brazil, Canada, China, Denmark, Finland, France, Germany, India, Ireland, Italy, Korea, Netherlands, Portugal, Spain, Taiwan, Turkey, Ukraine, United Kingdom) to support a Phase 3 study using a new drug product formulation without traditional longterm and accelerated data on the new formulation. The new formulation was the intended commercial formulation and was a formulated immediate release (IR) gelatin capsule. Phase 1 and 2 formulations included powder (drug substance) in a capsule (PIC) and IR tablet. Stability data for the Phase 1 and 2 formulations were supportive, since the data provided long-term data supporting chemical compatibility between the drug substance and most of the excipients being used in the capsule formulation.

A statistically designed 14-day ASAP study was performed on a development lot of the capsules that was compositionally equivalent to the proposed clinical capsules. The development lot was stored in open glass bottles and exposed to various temperatures, humidities and durations. Ranging from $50^{\circ} \mathrm{C} / 75 \% \mathrm{RH}$ to $80^{\circ} \mathrm{C} /$ $75 \% \mathrm{RH}$ and 5 to 14 days. Testing was performed on development batches containing the highest excipient to drug ratio for this product, maximizing possible drugexcipient interactions.

The stability samples were evaluated for appearance, levels of the main degradant, and total degradants. All appearance data remained within specification with no significant trends observed. The main degradant was determined to be the shelf life limiting degradant at room temperature conditions. The degradant was modeled, and the results were used to predict an initial clinical shelf life for the formulation. The model predicted an acceptable shelf life of at least 2 years, when stored at $30{ }^{\circ} \mathrm{C} / 75 \% \mathrm{RH}$.

The predictive results along with supportive data from the previous clinical formulations were filed to the countries listed above, along with a commitment to evaluate the stability of the clinical supplies through the duration of the clinical studies under long-term and accelerated conditions. The initial shelf-life was assigned as 12 months. This approach was accepted without queries in all countries except UK, Korea and Germany. Three months of long-term data were provided in response to queries in these countries and the shelf-life was approved. The ASAP predictions were subsequently confirmed by the available long-term stability data.

\section{Case studies: registration \\ Case study 16: justification of drug product strategy for water testing on stability}

For a recent immediate release formulation made up of an active-containing amorphous solid dispersion, it was determined that water content is not a critical quality attribute (CQA) and does not require analytical control. To justify this, in addition to a forced crystallization study and open dish chemical stability studies, dissolution was evaluated for the impact of temperature and water content. An ASAP study on tablet dissolution was designed using seven accelerated temperatures and humidity conditions ranging from $50{ }^{\circ} \mathrm{C} / 60 \% \mathrm{RH}$ to $60^{\circ} \mathrm{C} /$ $75 \% \mathrm{RH}$ for up to 2 weeks. Considerable changes in dissolution were observed under highly stressed conditions. The ASAP data along with data from the $40^{\circ} \mathrm{C} / 75 \% \mathrm{RH}$ open dish study at 3 and 6 months were used in the data modeling. With the fitted modified Arrhenius equation, along with the water sorption isotherms of the tablets and packaging configurations, dissolution profiles were predicted throughout the product shelf-life under any given storage condition and initial tablet water content. The model was then verified with available real time stability data and agreed with the observed real time data (Li et al. 2016).

The ASAP study and dissolution prediction model were presented in Section 3.2.P.2.3 Manufacturing Process Development of the product marketing application submitted to the USA and EU. The results demonstrated that under the proposed packaging and storage conditions the water content of the tablets would not impact dissolution justifying water testing is not required on stability. The data and strategy were accepted by the agencies with no related review queries. The ASAP predictions were subsequently confirmed by the available long-term stability data.

\section{Case study 17: justification of degradant end of shelf-life specification}

A Product Characterization Study (PCS) (Lavrich 2016; Wu et al. 2018) is a systematic study that explores the product stability as the product is exposed unprotected to various constant environmental conditions to understand the impact of storage conditions and formulation composition / manufacture process on the stability of product over the anticipated shelf life. It is used to build predictive stability models and to inform the design space, control strategy, specification setting, package selection, shelf-life and post-approval changes.

A PCS was performed to assess the impact of moisture and temperature on the degradation of an extended 
release tablet formulation over an anticipated 2-year shelf- life. The study was conducted under open dish conditions by exposing drug product stored at $25^{\circ} \mathrm{C}$, $30^{\circ} \mathrm{C}$ and $40{ }^{\circ} \mathrm{C}$ over a range of humidity conditions $(15 \%, 35 \%, 45 \%, 60 \%$ and $75 \% \mathrm{RH})$ with sampling at various time points over 2 years. PCS data revealed that the API in drug product was sensitive to moisture with hydrolysis degradation the primary degradation pathway. An example of relevant hydrolysis degradation results of the PCS study at $25^{\circ} \mathrm{C}$ is provided in Fig. 1 .

The data revealed that levels of the hydrolysis product were suitably controlled in samples stored below $35 \% \mathrm{RH}$. The selected high-density polyethylene (HDPE) bottle packages were designed to maintain a headspace $\mathrm{RH}$ of less than or equal to $35 \%$ for one potency and $30 \%$ for two other potencies over the course of 2 years when stored at room temperature conditions across all four climatic zones. The headspace moisture is effectively controlled in bottle packages by adjusting the desiccant loading to account for long-term moisture transmission across the primary package barrier, as well as the moisture introduced via the product mass.

To support the proposed shelf-life degradation limit, the PCS predictions were filed in NDA drug product sections 3.2.P.5.6 Justification of Specification(s) and 3.2.P.8.1 Stability Summary and Conclusion in multiple markets including the US, EU, Canada, Australia, Asia Pacific, and Latin America starting in 2011. The data and strategy were accepted by the agencies with no related regulatory queries.

\section{Discussion of regulatory feedback and regulatory trends}

Experience using risk-based predictive tools to set an initial clinical shelf-life reaches back to 2009. Overall, the regulatory acceptance of this strategy has been high. The collective experience of five companies involved with authoring this publication is summarized in Fig. 2. Here, the bars on the left (blue) represent the number of clinical applications that were submitted and approved utilizing only predictive stability to support the initial shelf-life. Within these submissions, commitments were also made to conduct long-term and traditional accelerated stability, but data from those studies were not available for the initial submission. The middle bars (red) represent the portion of those clinical submissions where the regulatory reviewer required submission of data from the traditional stability study prior to granting the requested shelf-life. The bars on the right (green) represent those submissions where the proposed shelf-life was not accepted. In the cases where the shelf-life was not accepted, the reasons provided link the non-acceptance to lack of long-term stability data, rather than relating the non-acceptance to specific concerns with the model used. Over the years, most of the regulatory applications that submitted only predictive data to support the initial shelf-life were accepted. As illustrated by the red bars in 2016 and 2017, the number of requests for additional data to substantiate the initial shelf-life appears to be increasing. And, although the contributing companies have not experienced the rejection of their application based

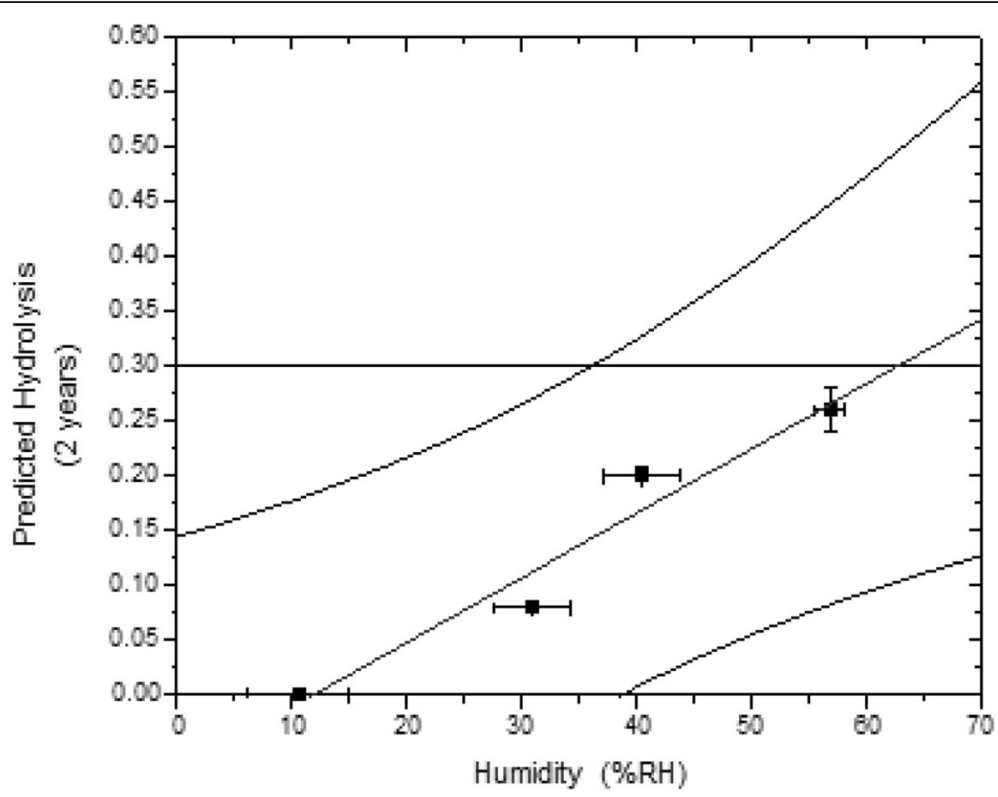

Fig. 1 Predicted Hydrolysis as a Function of Humidity at $25^{\circ} \mathrm{C}$. The Regression Line and the $95 \%$ Confidence Interval are Presented. The Proposed Specification Limit for the Hydrolysis Product is $0.30 \%$ 


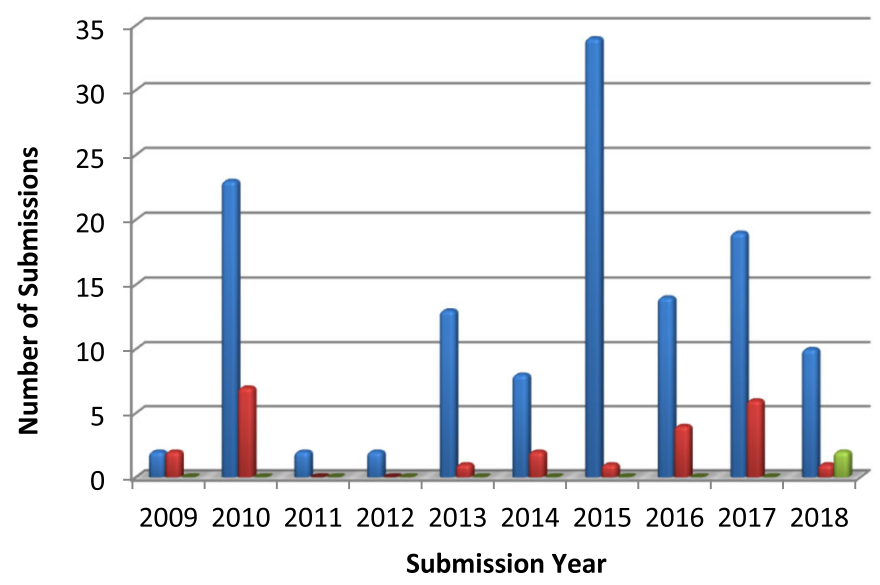

Accepted

Accepted After Provision

of Additional Data

$\square$ Shelf-life not accepted

Fig. 2 Regulatory Experience Using RBPS to Set an Initial Shelf-life

on this strategy, there are a small number of occurrences where the proposed shelf-life was not accepted.

There has been an apparent decrease in regulatory acceptance of the use of RBPS to set initial shelf-life since 2016. A higher percentage of submissions were queried for the provision of data from traditional studies. In 2018, some companies were not granted the requested shelf-life after the provision of long-term data or were not permitted the opportunity to provide additional data.
The recent trend may be in part due to the publication of the 2017 EMA clinical guideline (European medicines agency 2017) which provides increased instruction on how companies should set and extend shelf-life in the clinical stage and does not acknowledge RBPS as a tool to set an initial shelf-life. When using risk-based strategies to set or extend a clinical shelf-life that do not align with the recommendations in the guideline, companies should clearly articulate their justification.

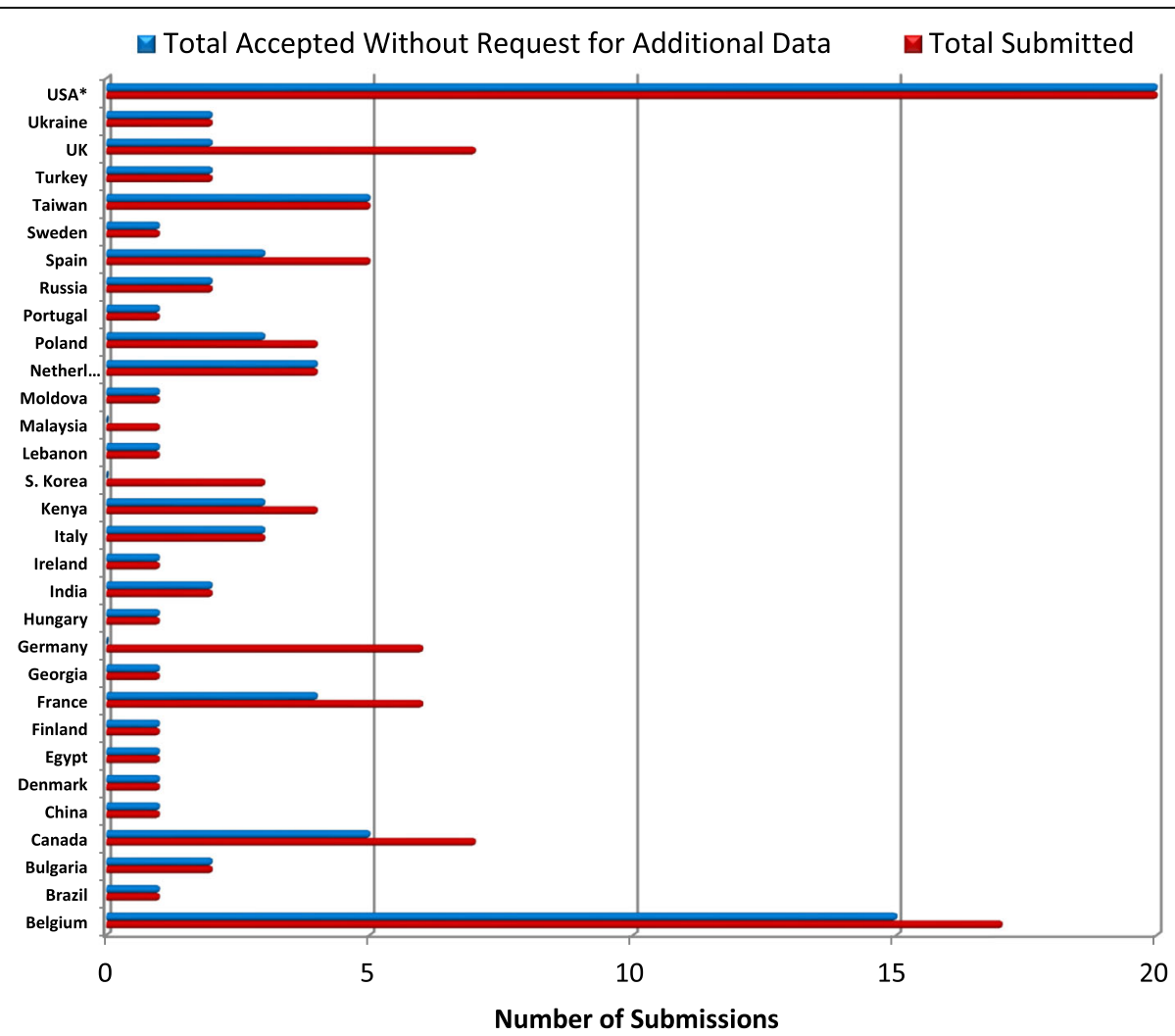

Fig. 3 RBPS Regulatory Experience by Country (2009-2018). *USA Bar is truncated (35 accepted without requests for additional data) 
The data since 2009 was also analyzed by country and these results are presented in Fig. 3. The bottom (red) bar represents the number of submissions made to a given country with only RBPS data for the proposed clinical material(s). The top (blue) bar represents the number of submissions that were approved without the provision of additional traditional stability data. The analysis indicates that RBPS approaches for setting initial shelf-life have generally been accepted by most countries. However, several countries do not embrace this approach. This could point to a need for further transparency and discussion within submissions on the details of the model and the assumptions that were made. Harmonization amongst industry on how these data are presented and utilized may also enable broader regulatory acceptance. A proposed approach for this was recently published (Stephens et al. 2018).

Beyond the clinical space, the case studies presented where RPBS data were used to support registration applications illustrate the broad range these tools have for impacting development knowledge. These case studies demonstrate how impactful RBPS data can be as supportive development data. Based on feedback received in the Industry Survey (Williams et al. 2017), companies are routinely using RBPS to identify optimal formulations, for packaging selection and to demonstrate equivalence to support changes. Discussion of these RBPS data within a marketing application would further underwrite fundamental product understanding.

\section{Conclusion}

The case studies presented in this paper demonstrate that RBPS can be and is being used to set an initial retest period for clinical drug substance, an initial shelf-life for many clinical formulations, and to support changes (e.g., to demonstrate equivalence after a process/formulation change or to assess the risk to stability after a packaging change) (Timpano et al. 2018). Where available, longterm data for each case are consistent with the RBPS predictions. The use of predictive tools to set initial retest/shelf-life is well-precedented and has been used routinely by some companies for almost ten years. It enables shelf-life to be set in a more expedient manner. This, in turn, enables submission of clinical applications in a timelier fashion. The regulatory risks to this approach are presented above and can be mitigated by ensuring the appropriate duration of long-term data is available for query responses to further substantiate the proposed shelf-life.

Analysis of the collective recent regulatory experience (refer to Fig. 2) suggests an apparent decrease in regulatory acceptance of the use of RBPS to set initial shelf-life since 2016. Companies with experience using these tools feel this is at odds with RBPS being a powerful and science-based tool for gaining a more rapid and thorough understanding of the stability characteristics of a drug substance and drug product. The depth of stability knowledge gained through an RBPS study typically well exceeds the information learned after, for example, the first 3-month time point of a long-term study where, for most well-designed products, changes are insignificant. Although the approach is not intended to replace longterm studies for setting shelf-life, it certainly complements traditional long-term and accelerated stability studies in that the modeling is ultimately validated by the long-term data.

The varied case studies presented herein demonstrate that the use of RBPS tools can play an important role in regulatory submissions. In the clinical space, RBPS can lead to more rapid development timelines which provides benefit for patients in need of new medications. The tools provide a key benefit to development and fundamental product understanding. There is a clear opportunity to continue to share this data and utilize it to convey enhanced product understanding in regulatory submissions. Beyond the case studies presented here, the potential regulatory applications for RBPS are many and diverse. Moving forward, these tools could be used to bring medicines to the market sooner, particularly when considering long-term (12 month) data per ICH can be the rate limiting step for a marketing application (European 2017). RBPS tools could be used effectively post-approval to understand and convey risk to stability after a change to a process or product, rather than relying on collection of long-term data (Malcom 2018). In these cases, RBPS once again has the potential to provide value adding and incisive information in a short amount of time. When used effectively, this data should enable industry to make the best decisions throughout development and lifecycle management and enhance regulatory reviewers' confidence in industry's abilities to understand the stability of our products and further protecting the patient.

\section{Abbreviations \\ ASAP: Accelerated Stability Assessment Program; HPMC: hydroxypropyl methyl cellulose; FIH: First in Human; ICH: International Council for Harmonization; IQ: International Consortium for Innovation and Quality; PCS: Product Characterization Study; PfOS: Powder for oral solution; RBPS: Risk-Based Predictive Stability}

\section{Acknowledgements}

Dennis Stephens, Abbvie.

\section{Authors' contributions}

All authors contributed case studies, ideas and information to assess regulatory feedback for this manuscript. HW authored the introduction to the manuscript. MM authored the discussion and conclusions. All authors reviewed and approved the manuscript.

\section{Funding}

Not applicable; authors contributed case studies based on existing company knowledge and experience. 
Availability of data and materials

Not applicable.

\section{Competing interests}

The authors declare that they have no competing interests.

\section{Author details}

${ }^{1}$ Pfizer Inc., Eastern Point Road, Groton, CT 06340, USA. ${ }^{2}$ AstraZeneca, Silk Road Business Park, Macclesfield, Cheshire SK10 2NA, UK. ${ }^{3}$ Janssen Pharmaceutica R\&D, a Division of Janssen Pharmaceutica NV, Turnhoutseweg 30, 2340 Beerse, Belgium. ${ }^{4}$ Sunovion Pharmaceuticals, 84 Waterford Dr, Marlborough, MA 01752, USA. ${ }^{5}$ Boehringer Ingelheim, 900 Ridgebury Road, Ridgefield, CT 06877, USA. ${ }^{6}$ Merck \& Co., Inc., Kenilworth, NJ 08550, USA. 7 Vertex Pharmaceuticals, 50 Northern Avenue, Boston, MA 02210, USA. ${ }^{8}$ Genentech, 1 DNA Way, South San Francisco, CA 94080, USA. ${ }^{9}$ Eli Lilly \& Company, Lilly Corporate Center, Indianapolis, IN 46285, USA. ${ }^{10}$ Biogen, 5000 Davis Dr, Durham, NC 27709, USA.

Received: 1 October 2019 Accepted: 17 April 2020

Published online: 11 May 2020

\section{References}

European federation of pharmaceutical industries and association (2017) EFPIAEBE White Paper on Expedited CMC Development: Accelerated Access for Medicines of Unmet Medical Need - CMC Challenges and Opportunities (Final Version - December 2017). https://www.efpia.eu/media/288657/efpiaebe-white-paper-expedited-cmc-development-accelerated-access-formedicines-of-unmet-medical-need-december-2017.pdf

European medicines agency (2017) EMA/CHMP/QWP/545525/2017 Guideline on the requirements for the chemical and pharmaceutical quality documentation concerning investigational medicinal products in clinical trials. https://www.ema.europa.eu/en/documents/scientific-guideline/ guideline-requirements-chemical-pharmaceutical-quality-documentationconcerning-investigational_en.pdf

Freed AL, Colgan ST, Kochling JD, Alasandro MS (2016) AAPS workshop: accelerating pharmaceutical development through predictive stability approaches, April 4-5, 2016. AAPS Open 3:8

ICH: Guideline Q10 (2008) Pharmaceutical quality system, Step 4 version. https:// www.ich.org/fileadmin/Public_Web_Site/ICH_Products/Guidelines/Quality/Q1 0/Step4/Q10_Guideline.pdf

ICH: Guideline Q11 (2012) Development and manufacture of drug substances (chemical entities and biotechnological/biological entities), step 4 version. https://www.ich.org/fileadmin/Public_Web_Site/ICH_Products/Guidelines/ Quality/Q11/Q11_Step_4.pdf

ICH: Guideline Q1A(R2) (2003) Stability testing of new drug substances and products, Step 4 version. https://www.ich.org/fileadmin/Public_Web_Site/ ICH_Products/Guidelines/Quality/Q1A_R2/Step4/Q1A_R2_Guideline.pdf

ICH: Guideline Q8(R2) (2009) Pharmaceutical development, step 4 version. https:// www.ich.org/fileadmin/Public_Web_Site/ICH_Products/Guidelines/Quality/ Q8_R1/Step4/Q8_R2_Guideline.pdf

ICH: Guideline Q9 (2005) Quality risk management, step 4 version. https://www. ich.org/fileadmin/Public_Web_Site/ICH_Products/Guidelines/Quality/Q9/ Step4/Q9_Guideline.pdf

Lavrich, D (2016) AAPS webinar: rapid development of robust stability models using semi-empirical design space, 24 March 24, 2016 https://aapsopen. springeropen.com/articles/10.1186/s41120-017-0018-5\#article-info

Li H, Nadig D, Kuzmission A, Riley CM (2016) Prediction of the changes in drug dissolution from an immediate-release tablet containing two active pharmaceutical ingredients using an accelerated stability assessment program (ASAP prime ${ }^{\oplus}$ ). AAPS Open 2:7

Malcom L (2018) Chapter 12: accelerated stability assessment Program (ASAP) applications in a postapproval environment. Accelerated predictive stability 1st edition pp:231-241. doi:https://doi.org/10.1016/C2014-0-02298-8

Oliva A, Faria JB, Llabrés M (2012) An improved methodology for data analysis in accelerated stability studies of peptide drugs: practical considerations. Talanta 94:158-166

Stephens D, Williams H, McMahon M, Qiu F, Hyzer CH, Debie E, Wu Y, Li H, Wang J (2018) Risk-based predictive stability for pharmaceutical development - a proposed regulatory template. Pharm Tech 42(8):42-47
Timpano RJ, Freed AL, Clement E, Masse K, Ryan K (2018) Chapter 9: accelerated predictive stability regulatory strategies. Accelerated predictive stability $1 \mathrm{st}$ edition pp:231-241. doi:https://doi.org/10.1016/C2014-0-02298-8

Waterman KC, Adami R (2005) Accelerated aging: prediction of chemical stability of pharmaceuticals. Int J Pharm 293(1-2):101-125

Waterman KC, Carella AJ, Gumkowski MJ, Lukulay P, MacDonald BC, Roy MC, Shamblin SL (2007) Improved protocol and data analysis for accelerated shelf-life estimation of solid dosage forms. Pharm Res 24(4):780-790

Wicks BS, Lewis T, Khawam A (2018) Chapter 15 applications of ASAP to generic drugs. Accelerated predictive stability $1^{\text {st }}$ edition pp: 341-352. doi:https://doi. org/10.1016/C2014-0-02298-8

Williams H, Bright J, Roddy E, Poulton A, Cosgrove SD, Turner F, Harrison P, Brookes A, MacDougall E, Abbott A, Gordon C (2018) A comparison of drug substance predicted chemical stability with ICH compliant stability studies. Drug Develop Ind Pharm 45(3):379-386

Williams H, Stephens D, McMahon M, Debie E, Qiu F, Hyzer CH, Sechler L, Orr R, Webb D, Wu Y, Hahn D (2017) Risk-based predictive stability - an industry perspective. Pharm Tech 41(3):52-57

Wu Y, McMahon M, Regler B (2018) Applications of accelerated stability models in product development. PharmSci360 short course. https://www. conferenceharvester.com/Uploads/Documents/HarvesterLink-5946-748(41 ).pdf

\section{Publisher's Note}

Springer Nature remains neutral with regard to jurisdictional claims in published maps and institutional affiliations.

\section{Submit your manuscript to a SpringerOpen ${ }^{\circ}$ journal and benefit from:}

- Convenient online submission

- Rigorous peer review

- Open access: articles freely available online

High visibility within the field

- Retaining the copyright to your article

Submit your next manuscript at $\boldsymbol{\nabla}$ springeropen.com 